\title{
From in silico astrocyte cell models to neuron-astrocyte network models: A review
}

Franziska Oschmann ${ }^{\mathrm{a}}$, Hugues Berry ${ }^{\mathrm{b}, \mathrm{c}}$, Klaus Obermayer $^{\mathrm{a}}$, Kerstin Lenk ${ }^{\mathrm{d}}$

Authors' affiliation addresses:

a Technical University Berlin, Neural Information Processing Group, Sekr. MAR 5-6, Marchstrasse 23, 10587 Berlin, Germany, oschmann@ni.tu-berlin.de b INRIA, 69603 Villeurbanne, France c LIRIS UMR5205, University of Lyon, 69622 Villeurbanne, France

d Tampere University of Technology, BioMediTech, PL100, 33014 Tampere, Finland, lenk.kerstin@gmail.com

Corresponding author: Kerstin Lenk, lenk.kerstin@gmail.com

\begin{abstract}
The idea that astrocytes may be active partners in synaptic information processing has recently emerged from abundant experimental reports. Because of their spatial proximity to neurons and their bidirectional communication with them, astrocytes are now considered as an important third element of the synapse. Astrocytes integrate and process synaptic information and by doing so generate cytosolic calcium signals that are believed to reflect neuronal transmitter release. Moreover, they regulate neuronal information transmission by releasing gliotransmitters into the synaptic cleft affecting both pre- and postsynaptic receptors. Concurrent with the first experimental reports of the astrocytic impact on neural network dynamics, computational models describing astrocytic functions have been developed. In this review, we give an overview over the published computational models of astrocytic functions, from single-cell dynamics to the tripartite synapse level and network models of astrocytes and neurons.
\end{abstract}

\section{Highlights}

- Astrocytes are active partners in neuronal communication.

- These glial cells integrate and process synaptic information.

- They regulate synaptic transmission by releasing neurotransmitters.

- We provide an overview over computational models of astrocytic functions.

Keywords: Neuron, Astrocyte, Tripartite synapse, Network, Simulation, In silico

$\begin{array}{ll}\text { Abbreviations: } \\ \text { AFM } & \text { (mixed) amplitude and frequency modulation } \\ \text { AM } & \text { amplitude modulation } \\ \text { AMPA } & \alpha \text {-amino-3-hydroxy-5-methyl-4-isoxazolepropionic acid } \\ \text { ATP } & \text { adenosine triphosphate } \\ \text { BC } & \text { basket cell } \\ \text { ER } & \text { endoplasmic reticulum } \\ \text { FM } & \text { frequency modulation } \\ \text { GABA } & \gamma \text {-Aminobutyric acid } \\ \text { GPCR } & \text { G-protein-coupled receptors } \\ \text { IP3 } & \text { inositol triphosphate } \\ \text { IP3R } & \text { IP3 receptor } \\ \text { IP3-3K } & \text { IP3 3-kinase } \\ \text { IP-5P } & \text { inositol polyphosphate 5-phosphatase } \\ \text { LTP/LTD } & \text { long-term potentiation/depression } \\ \text { mGluR } & \text { metabotropic glutamate receptors } \\ \text { NMDA } & N \text {-Methyl-D-aspartic acid } \\ \text { VGCC } & \text { voltage-gated Ca }{ }^{2+} \text { channels }\end{array}$




\section{Introduction}

In the last three decades, converging experimental reports have shown that astrocytes do not just provide structural support to the neurons. Astrocyte membrane extensions (processes) contact neuron and synapses. Through those contact sites, astrocytes are the main source of uptake / buffering of the molecules released by active neurons, including the neuronal glutamate released upon transmission of neuronal signal at excitatory synapses and the extracellular $\mathrm{K}^{+}$released by neuronal voltage-gated potassium channels during action potentials (Dallérac et al., 2013). But the interaction between astrocytes and neurons is not just about buffering of neuron biomolecules. First, astrocytes are thought to supply neurons with food (glucose) by providing them with glucose precursor lactate (Amiri et al. 2013; Kimelberg and Nedergaard 2010).

Moreover, astrocytes have consistently been implicated in the processing, transfer and storage of information by the nervous system (Perea and Araque 2010; McIver et al. 2013). In particular, astrocytes are involved in several forms of memory, including long-term plasticity (long-term potentiation/depression, LTP/LTD) (Gómez-Gonzalo et al., 2015; Henneberger et al., 2010) and neuronal excitability as well as synchrony (Clarke and Barres, 2013; Fellin, 2009).

Although the membrane of astrocytes is devoid of action potential, the concentration of calcium $\left(\mathrm{Ca}^{2+}\right)$ in the astrocyte cytoplasm is considered to represent an active signaling mechanism: cytosolic $\mathrm{Ca}^{2+}$ transients display a repertoire of dynamics, some of which have been identified as a response to neuronal activity (Figure 1). Astrocytes express on their plasma membrane the molecular equipment (channels, receptors, signaling) needed to detect the neuroactive molecules released by the neurons when they fire action potentials and encode this signal into the dynamics of their cytosolic $\mathrm{Ca}^{2+}$ (Dallérac et al., 2013). Recent reports tend to organize these various $\mathrm{Ca}^{2+}$ signals into three categories, depending on their spatio-temporal scale (Di Castro et al., 2011; Srinivasan et al., 2015): small-amplitude rapid and localized transients restricted to the process ends; larger locally-propagating calcium waves; and large, slow calcium transients encompassing the whole cell, including its cell body. However, it is not yet clear if these signals are generated by a unique underlying molecular mechanism nor what category provides the response to neuronal activity.

At glutamatergic synapses, a widely documented signaling pathway connecting neuronal activity to cytosolic $\mathrm{Ca}^{2+}$ in the astrocyte goes through metabotropic glutamate receptors (mGluR). After a sequence of incoming spikes at the presynapse, glutamate is released to the synaptic cleft and part of it binds to group I mGluRs in the astrocyte plasma membrane. These $\mathrm{G}_{\mathrm{q}}$-linked G-protein-coupled receptors (GPCR) activate PLC $\beta$, which in turn elevates the level of second messenger inositol triphosphate (IP3). IP3 sensitizes $\mathrm{Ca}^{2+}$-specific IP3 receptor channels (IP3Rs) in the membrane of the endoplasmic reticulum (ER), resulting in the release of $\mathrm{Ca}^{2+}$ from the ER to the cytoplasm (Petravicz et al., 2008).

In analogy with neurons, astrocytes also release neuroactive biomolecules (called gliotransmitters, in analogy with neurotransmitters) including glutamate, D-serine, adenosine triphosphate (ATP), and GABA ( $\gamma$ aminobutyric acid; Figure 1). Released gliotransmitters have been evidenced to influence neuronal function, with potential excitatory or inhibitory effects (De Pittà et al., 2012). Several mechanisms have been described for gliotransmitter release, including more or less neuron-like exocytosis vesicles, membrane channels or pumps. But in many instances (e.g. for D-Serine, glutamate and ATP) the release is triggered by elevation of cytosolic $\mathrm{Ca}^{2+}$ (Henneberger et al., 2010; Pasti et al., 2001; Sahlender et al., 2014; Zorec et al., 2012). Taken together, the above results support bidirectional communication between the neuron and the astrocyte. The concept of the "tripartite synapse" was introduced by Araque, et al. (1999) to coin the assembly formed by the astrocyte and the connected pre- and postsynapse it enwraps, as well as the bidirectional signaling they may thus support.

Astrocytes too form interconnected networks that can transmit signal under the form of intercellular calcium waves (Scemes and Giaume 2006). The propagation of these waves can be supported by two pathways: direct cytosol-to-cytosol transfer of IP3 via gap-junctions connecting the cells or paracrine ATP signaling (ATP release from the emitter cell, extracellular diffusion and binding to the receiver cell). Both direct IP3 transfer and ATP binding lead to transient $\mathrm{Ca}^{2+}$ elevation in the receiver cell, thus supporting wave propagation. However, it is not known how the local rapid $\mathrm{Ca}^{2+}$ transients evoked above interact with (or integrate into) the $\mathrm{Ca}^{2+}$ wave of intercellular propagation.

Numerous reports have implicated astrocytes in various central nervous system disorders, like multiple sclerosis (Brosnan and Raine, 2013), epilepsy (Seifert and Steinhäuser, 2013), Alzheimer's (Vincent et al., 2010) and Parkinson's disease (Maragakis and Rothstein, 2006; Rappold and Tieu, 2010).

Parallelizing the increasing number of experiment reports that investigate the role of astrocytes in health and pathological conditions, several computational or mathematical models have also been published, focusing on different scales: single cell astrocyte models, tripartite synapse models or neuron-astrocyte network 
models. The early computational models, relating either to normal or pathological contexts, have already been surveyed in review articles (Volman et al. 2012; Linne and Jalonen 2014). However, in the last three years, new substantial efforts have been made to model neuron-astrocyte interactions in the tripartite synapse and on network level. Our objective in this review is to give an updated overview of the computational and mathematical models of astrocytes, from in silico single cell models to neuron-astrocyte network models. We present and discuss the in silico models at these three scales (see Table 1 for an overview of astrocyte models with increasing complexity) and present the corresponding approach to the simulation of neurodegenerative disease whenever applicable. We focus on the role of astrocytes in the modulation of neuronal information transmission and plasticity and do not attempt to describe the models dedicated to the implication of astrocytes in metabolic support of the neurons (e.g., Aubert and Costalat, 2005; Jolivet et al., 2009) nor on vascular coupling (e.g., Chander and Chakravarthy, 2012; Zheng et al., 2010).

Figure 1: Scheme of the tripartite synapse: Incoming spikes from the presynapse lead to a release of neurotransmitters in the synaptic cleft. These transmitters are taken up by astrocytic receptors such as mGluRs which trigger IP3 release. Calcium is released from the endoplasmic reticulum which in turn leads to gliotransmitter release from the astrocyte. Glutamate and ATP bind to the corresponding receptors at the presynapse and GABA, glutamate and D-serine bind to the receptors at the postsynapse which modulates the firing rate. Astrocytes are connected to each other via gap junctions which are permeable for IP3 and thus promote calcium wave propagation.

Table 1: Overview of astrocyte models with increasing complexity (second column). The third column shows the number of neurons $(\mathrm{N})$ and the number of astrocytes (A). The fourth column indicates whether IP3 and/or calcium dynamics were modelled. The last column specifies the involved gliotransmitters ( $\mathrm{Glu}=$ glutamate; $\mathrm{ATP}=\mathrm{adenosine}$ triphosphate; $\mathrm{ADP}=$ adenosine diphosphate; $\mathrm{UTP}=$ uridine-5'-triphosphate; $\mathrm{UDP}=$ uridine diphosphate; $\mathrm{NS}=; \mathrm{GABA}=$ $\gamma$-Aminobutyric acid).

\begin{tabular}{|l|l|l|l|l|}
\hline Model & Cell/network & $\begin{array}{l}\text { Model size } \\
\text { \# neurons, } \\
\# \text { astrocytes) }\end{array}$ & $\begin{array}{l}\text { IP3/ } \\
\text { Ca2+ }\end{array}$ & Gliotransmitter \\
\hline Goldbeter et al., 1990 & Astrocyte only & A: 1 & both & Agonist (not defined) \\
\hline Young \& Keizer, 1992 & Astrocyte only & A: 1 & both & Agonist (not defined) \\
\hline Dupont and Goldbeter, 1993 & Astrocyte only & A: 1 & both & Agonist (not defined) \\
\hline Li \& Rinzel, 1994 & Astrocyte only & A: 1 & both & Agonist (not defined) \\
\hline
\end{tabular}




\begin{tabular}{|c|c|c|c|c|}
\hline Goto et al., 2004 & Astrocyte only & A: 1 & both & Glu \\
\hline Larter \& Craig, 2005 & Astrocyte only & A: 1 & both & Glu \\
\hline Stamatakis and Mantzaris, 2006 & Astrocyte only & A: 1 & both & ATP \\
\hline De Pittà et al, 2009 & Astrocyte only & A: 1 & both & Glu \\
\hline Zeng et al., 2009 & Astrocyte only & A: 1 & both & Agonist (not defined) \\
\hline De Pittà et al., 2011 & $\begin{array}{l}1 \text { Presynapse + } \\
1 \text { Astrocyte }\end{array}$ & $\mathrm{N}: 1, \mathrm{~A}: 1$ & both & Glu \\
\hline Mesiti et al., 2015 & $\begin{array}{l}1 \text { Presynapse }+ \\
1 \text { Astrocyte }\end{array}$ & $\mathrm{N}: 1, \mathrm{~A}: 1$ & both & Glu \\
\hline Nadkarni et al., 2007 & Tripartite synapse & $\mathrm{N}: 2, \mathrm{~A}: 1$ & both & Glu \\
\hline Postnov et al., J Biol Phys, 2008 & Tripartite Synapse & $\mathrm{N}: 2, \mathrm{~A}: 1$ & both & Glu \\
\hline Valenza et al., 2011 & Tripartite sysnapse & $\mathrm{N}: 2, \mathrm{~A}: 1$ & both & Glu \\
\hline Wade et al., 2011 & $\begin{array}{l}\text { Tripartite synapse/ } \\
\text { Network }\end{array}$ & $\mathrm{N}: 2-4 ; \mathrm{A}: 1$ & both & Glu \\
\hline Tewari and Majumdar, 2012 & Tripartite synapse & $\mathrm{N}: 2, \mathrm{~A}: 1$ & both & Glu \\
\hline De Pitta and Brunel, 2016 & Tripartite synapse & $\mathrm{N}: 2, \mathrm{~A}: 1$ & both & Glu \\
\hline Höfer et al, 2002 & Astrocyte network & A: $1-400$ & both & Not specified \\
\hline Bellinger et al. 2005 & Astrocyte network & A: 9 & both & Glu, ATP \\
\hline Iacobas et al., 2006 & Astrocyte network & A: $1-625$ & $\mathrm{Ca} 2+$ & ATP, ADP, UTP, UDP \\
\hline Ullah et al., 2006 & Astrocyte network & A: 2 & both & Glu \\
\hline Kang and Othmer, 2009 & Astrocyte network & A: 11 & both & Glu, ATP \\
\hline Goldberg et al., 2010 & Astrocyte network & A: $3-100$ & both & Not applicable \\
\hline Matrosov and Kazantsev, 2011 & Astrocyte network & A: 3 & both & Not mentioned \\
\hline Wei and Shuai, 2011 & Astrocyte network & A: 169 & both & Not mentioned \\
\hline Lallouette et al, 2014 & Astrocyte network & A: 1300 & both & Not mentioned \\
\hline Edwards and Gibson, 2010 & \begin{tabular}{|l|} 
Astrocytes and \\
Müller cells \\
\end{tabular} & A: 361 & both & ATP \\
\hline Postnov et al., 2009 & Network & $\begin{array}{l}\mathrm{N}: 2-3 ; \mathrm{A}: 1 \text { or } \\
\mathrm{Ca}^{2+} \text { syncytium }\end{array}$ & both & Glu, ATP \\
\hline Sotero and Martínez-Cancino, 2010 & Network & $\begin{array}{l}500,000 \text { tripartite } \\
\text { synapses }\end{array}$ & both & Glu \\
\hline Amiri et al., 2013 & Network & N: $100 ; A: 50$ & both & Glu, ATP \\
\hline Tewari and Parpura, 2013 & Network & $\mathrm{N}: 5, \mathrm{~A}: 1$ & both & Glu \\
\hline Valenza et al., 2013 & Network & N: $1000, A: 1500$ & both & Glu \\
\hline Naeem et al., 2015 & Network & $\mathrm{N}: 2-10 ; \mathrm{A}: 1-5$ & both & Glu, (endocannabinoids) \\
\hline Yang \& Yeo 2015 & Network & N: $512 ; A: 28$ & both & Glu \\
\hline Lenk et al., 2016 & Network & N: 250; A: 107 & both & Glu, GABA, ATP \\
\hline
\end{tabular}

\section{Single astrocytic cell models}

\section{$2.1 \mathrm{Ca}^{2+}$ signals in astrocytes}

Just like in several other cell types, the dynamics of free cytosolic calcium is central to astrocyte physiology, either for astrocyte-astrocyte communication or for astrocyte-neuron interactions. In astrocytes, $\mathrm{Ca}^{2+}$ dynamics are usually considered as resulting from $\mathrm{Ca}^{2+}$ flows between the cytoplasm and IP3-sensitive internal calcium stores although the importance of exchanges with extracellular $\mathrm{Ca}^{2+}$ is not excluded (see e.g. Shigetomi et al. (2012). At glutamatergic synapses for instance, the glutamate released by the presynaptic neuron activates group I mGluRs in the astrocyte plasma membrane. These mGluRs are $\mathrm{G}_{\mathrm{q}}$-linked GCPRs that activate PLC $\beta$, thus increasing the amount of IP3. This IP3 surge opens $\mathrm{Ca}^{2+}$-specific IP3R channels in the membrane of the ER, resulting in an elevation of $\mathrm{Ca}^{2+}$ in the cytoplasm (Petravicz et al., 2008). Whereas the early mathematical descriptions of the IP3- and $\mathrm{Ca}^{2+}$ - dependent release of $\mathrm{Ca}^{2+}$ from IP3R-gated stores from De Young-Keizer and Li-Rinzel (De Young and Keizer, 1992; Li and Rinzel, 1994) has been adopted in most subsequent models for $\mathrm{Ca}^{2+}$ dynamics in astrocytes (De Pittà et al., 2009; Goto et al., 2004; Höfer et al., 2002), the description of IP3 dynamics in the models is more heterogeneous. In the early models (Goldbeter et al. 1990; Dupont and Goldbeter 1993; Li and Rinzel 1994), the concentration of IP3 is a 
parameter, not a time-dependent variable. These models predict the occurrence of self-sustained $\mathrm{Ca}^{2+}$ oscillations thus indicating that sustained $\mathrm{Ca}^{2+}$ oscillations do not require periodic variations of IP3. Yet, IP3 dynamics, too, are crucial in astrocytes since IP3 formation and consumption is Ca-dependent and IP3 exchange between two coupled astrocytes supports a large part of astrocyte communication. The model proposed by De Young and Keizer, 1992 already accounted for IP3 transients as a result of PLC $\beta$ activation by an external agonist (e.g. glutamate). A decade later, two new models featured a more complete description of IP3 dynamics, supplementing agonist-dependent IP3 synthesis with $\mathrm{Ca}^{2+}$-dependent, agonistindependent IP3 synthesis and IP3 transfer between astrocytes (Höfer et al., 2002; Goto et al., 2004). Indeed, the production of IP3 is also boosted by elevations of cytosolic $\mathrm{Ca}^{2+}$ concentration, via the $\mathrm{Ca}^{2+}$-activated PLC $\delta$ isoform (Venance et al., 1997). Importantly, $\mathrm{Ca}^{2+}$-dependent IP3 synthesis by PLC $\delta$ de facto induces a positive feedback between $\mathrm{Ca}^{2+}$ and IP3 leading to sustained oscillations of IP3 that are synchronous with the oscillations of $\mathrm{Ca}^{2+}$ (Goto et al., 2004). Finally, realistic $\mathrm{Ca}^{2+}$-dependent dynamics for IP3 synthesis and degradation was proposed by De Pittà et al. (2009). Here, IP3 degradation is mediated by two pathways: through phosphorylation of IP3 by inositol polyphosphate 5-phosphatase (IP-5P) and through phosphorylation of IP3 by IP3 3-kinase (IP3-3K), which is $\mathrm{Ca}^{2+}$ dependent (De Pittà et al., 2009). $\mathrm{Ca}^{2+}-$ dependent IP3 degradation by IP3-3K competes with $\mathrm{Ca}^{2+}$-dependent IP3 synthesis by PLC $\delta$, yielding a rich repertoire of dynamical regimes, in particular concerning how changes of extracellular glutamate are encoded into Ca and IP3 oscillations.

The early mathematical models for IP3R channel dynamics (De Young and Keizer 1992; Li and Rinzel 1994) are based on steady-state experimental data, e.g. the influential paper by Bezprozvanny, Watras, and Ehrlich (1991). From these data, mathematical models could be set to reproduce the mean of the measured statistics (at best, its distribution), over a long time or a large population of channels. Recently however, the availability of single-channel IP3R kinetics in vivo fostered the development of new types of models that are able to correctly reproduce the whole opening statistics of a single IP3R channel (Cao et al., 2014, 2013; Siekmann et al., 2012). Since these models are adapted to the study of low numbers of IP3R molecules, they may lead to new insights on e.g., the multiscale generation of intracellular calcium dynamics (Cao et al., 2013). However, to our knowledge, they have not yet been applied to the specific context of astrocytes and neuron-astrocyte interactions.

\subsection{Encoding mode of the agonist}

A consistent prediction of the above models is the appearance of cytosolic calcium oscillations in response to extracellular agonist application. The models predict that changes in agonist concentrations result in alterations either of the amplitude or of the frequency of the $\mathrm{Ca}^{2+}$ oscillations (Dupont and Goldbeter 1993; De Pittà et al. 2009). The common interpretation is that cytosolic calcium encodes the level of extracellular agonists by modulation of their oscillation amplitude (Amplitude Modulation, AM) or frequency (Frequency Modulation, FM). Prevalence of AM or FM encoding depends on the type of bifurcation giving rise to the oscillations (e.g. Hopf versus Saddle-Node on Invariant Circle), i.e. on the values of a handful of parameters (De Pittà et al., 2009). Note however that the potential existence of two output oscillators, $\mathrm{Ca}^{2+}$ and IP3, possibly partially uncoupled, could endow the system with more complex encoding modes, including mixed Amplitude and Frequency modulation (AFM, De Pittà et al., 2009). In astrocytes, available experimental data rather point to FM encoding albeit AM or AFM cannot be ruled out. The point is however especially relevant to astrocytes since it has been consistently reported that the release of gliotransmitters is triggered when cytosolic $\mathrm{Ca}^{2+}$ level exceeds a threshold value (see De Pittà et al. (2012) for a discussion). In this case then, pure FM encoding without amplitude modulation would lead to very different signaling compared to e.g. AM encoding.

\section{3 $\mathrm{Ca}^{2+}$ influx from extracellular space}

In most experimental reports, $\mathrm{Ca}^{2+}$ dynamics in astrocytes are mainly conditioned by flows between the cytoplasm and intracellular stores. However, $\mathrm{Ca}^{2+}$ influx from the extracellular compartment to the cytosol has also been evoked (see e.g. Shigetomi et al. (2012)). Accordingly, some of the detailed models also consider the influx of $\mathrm{Ca}^{2+}$ from the extracellular space through $\mathrm{Ca}^{2+}$ channels. In most cases $\mathrm{Ca}^{2+}$ entry is mediated in those models by voltage-gated $\mathrm{Ca}^{2+}$ channels (VGCC) with Hodgkin-Huxley equations (Li et al., 2012; Postnov et al., 2008; Zeng et al., 2009). In particular, Zeng and colleagues related the functional role of VGCCs to spontaneous $\mathrm{Ca}^{2+}$ oscillations in astrocytes and found out that $\mathrm{Ca}^{2+}$ oscillations are restricted to specific ranges of the membrane voltage and the intracellular $\mathrm{Ca}^{2+}$ concentration (Zeng et al., 2009).

\subsection{Gliotransmission}


In most models, the gliotransmitter released by the astrocyte impinges on the (pre- or post-synaptic) neurons, but not on the very astrocyte that released the gliotransmitter, thus defining the tripartite synapse models that will be addressed below. A notable exception is the model proposed by Larter and Craig, 2005, in which the glutamate released by the modelled astrocyte acts on the releasing astrocyte in addition to the glutamate released by the neurons. This model predicts that such a glutamate feedback should trigger complex bursting oscillations of cytosolic calcium and extracellular glutamate in response to background neuronal stimulation (Larter and Craig 2005).

Experimental studies have shown that the release of ATP by astrocytes plays a key role in wave propagation in astrocytic cellular networks. However, it is not clear yet if the release of ATP is $\mathrm{Ca}^{2+}$ or IP3 dependent.

For that purpose, Stamatakis and Mantzaris implemented both mechanisms and compared the resulting $\mathrm{Ca}^{2+}$ dynamics. Whereas in the $\mathrm{Ca}^{2+}$-dependent case astrocytes exhibited excitable behavior and frequency-

encoded oscillations, one expects one- and two-dimensional wave patterns with finite range of propagation in the IP3-depdent case (Stamatakis and Mantzaris, 2006).

\section{Tripartite synapse models}

\subsection{Tripartite synapse models concentrating on voltage-gated calcium channels}

A first support for information transfer between neurons and astrocytes consists in changes in the extracellular ion concentrations caused by neuronal activity. During high frequency firing of neurons, potassium accumulates in the extracellular space. The increased potassium concentration depolarizes the glia cells and voltage-dependent $\mathrm{Ca}^{2+}$ channels open.

Nadkarni and Jung (2007) base their model on the Pinsky and Rinzel model (1994) which is a 2compartment, eight-variable model of a CA3 pyramidal cell as a reduction of the complex 19-compartment cable model by Traub, et al. (1991) and the Li-Rinzel model for calcium dynamics in the astrocyte. Nadkarni and Jung simulated a tripartite synapse including the modulation of synaptic transmission mediated by the astrocyte and the spontaneous postsynaptic events which depend on astrocytic calcium signaling. In this model, the glutamate released by the astrocyte binds to metabotropic glutamate receptors (mGluRs) at the presynaptic membrane. The resulting increase of the $\mathrm{Ca}^{2+}$ concentration in the presynapse controls the release probability. The authors suggested that long-term facilitation at the synapse is due to the astrocytic glutamate release which leads to an increase of the calcium concentration in the presynapse. In a follow up study, the same group has suggested that astrocytes optimize synaptic information transmission by controlling the competition dynamics between induced and asynchronous (spontaneous) vesicle release in the neurons (Nadkarni et al., 2008).

Postnov and colleagues showed that this information transfer between neurons and astrocytes conveyed by potassium is part of a positive feedback loop causing the self-sustained activity of the postsynaptic neuron and prolonged activity of the glial cell (Postnov et al., 2008).

Another tripartite synapse model was proposed by Amiri et al. (2011) by connecting two gap-junction coupled Morris-Lecar neuron models (pre and postsynaptic) with the astrocyte model of Postnov et al. (2009). In the absence of the astrocyte, the activity of the two neurons was synchronized. Introduction of the astrocytes desynchronized the two coupled neurons. Simulation of defective astrocytic functions was introduced as a weakening of the impact of gliotransmitters on the neurons. In this pseudo-pathological case, the neuronal activity synchronized back over time.

\subsection{Tripartite synapse models concentrating on potassium dynamics}

The approach of including neuronal and astrocytic potassium dynamics into tripartite synapse models has also been used to study the uptake of accumulating extracellular potassium by astrocytes during neuronal excitation (potassium buffering). These models either focus on the neuronal dynamics while describing the astrocytic dynamics by simplistic models which only account for glial potassium uptake (Kager et al., 2007, 2002, 2000; Somjen et al., 2008), or they focus on both neuronal and astrocytic dynamics and also describe astrocytic membrane transport mechanisms (Øyehaug et al., 2012). During high extracellular potassium concentration, neurons become inactivated due to membrane depolarization. Astrocytes counteract this phenomenon by uptaking potassium from the extracellular space. Øyehaug and colleagues showed that a reduction of glial potassium transport mechanisms is expected to promote neuron inactivation (Øyehaug et al., 2012).

\subsection{Tripartite synapse models concentrating on gliotransmission}

Astrocytes may first affect synaptic transmission by uptaking glutamate from the synaptic cleft. Indeed, modulations of glutamate dynamics in the synaptic cleft alter the response of ionotropic AMPA and NMDA 
receptors at the postsynaptic membrane. Several computational studies investigate the amount of released glutamate (Savtchenko et al., 2013) and the glutamate time course in the synaptic cleft. The glutamate time course depends on the geometry and the transporter efficiency (Kinney et al., 2013). A particular emphasis is put on the glutamate transporters coverage in the synaptic cleft and how that affects glutamate diffusion into extrasynaptic regions (Rusakov, 2001; Zheng et al., 2008). For example, Diamond and colleagues discovered that a slower glutamate clearance in younger animals allows glutamate to diffuse longer distances (Diamond, 2005). In addition, the effect of astrocytic glutamate uptake is more efficient for sufficiently long inter-spike intervals in order to allow receptor recovery from desensitization (Allam et al., 2012).

Another source of regulation of synaptic transmission by the astrocyte is gliotransmission. The potential effects of gliotransmission on presynaptic short-term plasticity have been evaluated by a model in De Pittà et al. (2011), and, the effect of gliotransmission on postsynaptic calcium-controlled spike-timing dependent plasticity by the model of De Pittà and Brunel (2016). These works showed that calcium-dependent release of gliotransmitters by the astrocytes can switch synaptic plasticity between depression and potentiation regimes, depending in particular on the repertoire of receptors expressed at the presynaptic and postsynaptic terminals.

Tewari and Majumdar (2012) confirmed with their computational model that astrocytic calcium can contribute to short-term potentiation of the synapse and they identified two important parameters in the system: the releasable pool size of the synaptic-like micro-vesicles of the astrocyte and the maximum rate of IP3 production.

\subsection{Tripartite synapse models concentrating on learning}

Several experimental studies give evidence that astrocytes play a role in LTP/LTD (Gómez-Gonzalo et al. 2015; Henneberger et al. 2010; Paixão and Klein 2010). Wade, et al. (2011) combined the gatekeeper model (Volman et al., 2007) and the Nadkarni and Jung model $(2004,2007)$. Their results suggest that the extrasynaptic slow inward calcium currents triggered by gliotransmitter release can control plasticity and synchronous activity in the postsynapse.

\subsection{Tripartite synapse models concentrating on electrical descriptions}

Some models use a more electrical engineering approach to describe the tripartite synapse. Valenza et al. (2011) used an Izhikevich neuron model, the Nadkarni and Jung model (2004) for intracellular IP3 dynamics and the Li-Rinzel model (1994) for intracellular $\mathrm{Ca}^{2+}$ concentration, to create a computationally efficient model with nonlinear transistor-like transfer function, linking the parts of the tripartite synapse which they call "dressed neuron".

Mesiti, et al. (2015) base their engineering approach on the Li-Rinzel model (1994) and divide the presynapse-astrocyte pathway into input/output circuits. As De Pittà et al. (2009), they are able to modify the parameters of IP3 to evoke amplitude and frequency modulation of $\mathrm{Ca}^{2+}$. Their stimulus-response analysis explores how varying the astrocyte stimulations changes the presynaptic calcium levels. Furthermore, they indicate that some stimulation patterns elicited by the astrocytes may be more effective than others to change the neuronal activity.

\section{Astrocyte network models}

The coupling of astrocytes via gap junctions and their permeability to e.g. IP3 allows intercellular communication between astrocytes, which also affects the neuroglial and gliovascular interaction (Giaume et al., 2010). When the coupling coefficient between two astrocytes is within a certain range, the cytosolic $\mathrm{Ca}^{2+}$ of coupled astrocytes synchronize in anti-phase whereas it oscillates in phase beyond this range (Ullah et al., 2006). For coupling coefficients below this range, astrocytes oscillate with different frequencies and phases. Ullah et al. (2006) found only few astrocytes in anti-phase in their experimental data, they thus used distributed values of the maximum IP3 production for each cell as a way to expresses heterogeneity in the gap junction coupling.

Matrosov and Kazantsev (2011) investigate calcium oscillations in a small astrocyte network using bifurcation analysis. Random spontaneous $\mathrm{Ca}^{2+}$ events might be an effect of astrocyte connectivity combined with certain chaotic attractors. Wei and Shuai (2011) found a large regime of bistable calcium dynamics in their model which could not be observed in other models.

The propagation of intercellular calcium waves in model astrocytes networks was studied first with small linear astrocyte chains (Goldberg et al., 2010) and later extending the scope to large three-dimensional astrocyte networks of various topologies (Lallouette et al., 2014). The main conclusions from these articles are that long-range propagation of $\mathrm{Ca}^{2+}$ waves in astrocyte networks via direct IP3 transfer necessitates 
nonlinear transport of IP3 through the gap junctions and sparsely connected astrocyte networks. Moreover, they proposed a highly simplified model for the intracellular Ca-IP3 dynamics that preserves network propagation properties. Parts of these model predictions were validated against in vitro experimental results in Wallach et al. (2014).

To simulate experimental data in an adequate way, the model of Edwards and Gibson (2010) required two pathways in the astrocyte: intercellular through gap junctions and extracellular via ATP release. When gap junctions were added to the extracellular model, the speed of calcium propagation increased. By including Müller cells, the model was applied to simulate experimental results from the retina.

Bellinger (2005) studied the $\mathrm{Ca}^{2+}$ wave propagation mediated by the extracellular signals ATP and glutamate. ATP and glutamate both evoke calcium influx into the astrocyte and by that contribute to the generation of calcium waves. They observed that while a change of the intracellular IP3 concentration affects the initial rise of the amplitude, a change of the glutamate receptor affinity affects the duration of the calcium wave (Bellinger, 2005). Iacobas et al. (2006) investigated the spread of intercellular $\mathrm{Ca}^{2+}$ waves mediated by both intracellular and extracellular mechanisms. Whereas the intracellular mechanism was mediated by IP3, the extracellular mechanism was based on the release of ATP and other nucleotides, which activate $\mathrm{Ca}^{2+}$ mobilization by binding to membrane receptors or evoke $\mathrm{Ca}^{2+}$ influx. Their model serves as a tool for analyzing the expression level of receptors or the coupling of astrocytes via gap junctions under pathological conditions (Iacobas et al., 2006). Also Kang and colleagues studied the spatiotemporal characteristics of calcium waves in small-size astrocyte networks mediated by both intracellular (IP3) and extracellular (ATP) signals (Kang and Othmer, 2009). They applied their model to a complex realistic geometry with large areas of cell free domains. The simulation results revealed different characteristics of the intracellular and the extracellular signal concerning their propagation speed, propagation distance and the delay between cells. Both the IP3 and the ATP signal had a low propagation speed and they only propagated for a short distance. Whereas there was only a short delay between the cells for the IP3-signal, the delay time between the cells was much longer for the ATP-signal. This has especially a huge impact in the realistic geometry. The large area of cell free domains led to a rapid decay of the ATP signal and the concentration remained close to the steady state level (Kang and Othmer, 2009).

\section{Neuron-astrocyte network models}

The first model to quantify and include astrocytic activity on neuronal dynamics stems from Nadkarni and Jung (2003). In this paper, the authors describe the minimal model including a neuron with a single compartment and astrocytes as a homogeneous reservoir.

Valenza et al. (2013) extended their transistor-like description of the tripartite synapse (see section 3.5) with the addition of short-term synaptic plasticity for excitatory synapses. They simulated a network containing 1,000 neurons and 1,500 astrocytes where each astrocyte can be connected to several synapses. The model is able to produce spontaneous polychronous activity, i.e. reproducibly time-locked but not synchronous, in neural groups.

Savtchenko and Rusakov (2014) consider a ring network of hippocampal basket-cell-like (BC) neurons driven by excitatory input and monitor the synchronization of the BC. They compare the effect of changes of the excitatory drive when this input is randomly (uniformly) spread over the network or when it is clustered in space to a neighborhood of BC. The latter represents the effect of a local astrocyte network that releases gliotransmitters locally and locally increases extrasynaptic/ tonic gliotransmitter.

Neuron-astrocyte network models have also been devised to study the potential role of astrocytes on network robustness. When the communication between synapses fail for example due to neurodegenerative diseases, astrocytes could modulate IP3 diffusion via gap junctions to maintain $\mathrm{Ca} 2+$ oscillations and subsequently the global endocannabinoid- mediated synaptic potentiation signal in a syncytia (Perea and Araque 2005; Wade et al. 2012). After introducing B-STDP learning rule into the model, which combines spike-timingdependent plasticity and Bienenstock, Cooper, and Munro learning rules, the firing rate of neurons with damaged synapses recovers over time (Naeem et al., 2015).

Amiri et al. (2013) extended their model (see section 3.1) to 50 pyramidal neurons, 50 interneurons and 50 astrocytes, connected in a chain-like manner. Inhibitory and excitatory neurons were connected by pairs to their neighboring cells. Each astrocyte was connected with one pyramidal cell and one interneuron as well as with one neighboring astrocyte via gap junctions. In this model, increasing the influence of the astrocytes on the neurons leads to a reduction of synchronized neuronal oscillations.

Experiments on the CA3/Schaffer collateral-CA1 pyramidal neuron pathway suggest that astrocytes contribute to contextual memory retrieval and thus the synchronization of the CA1 pyramidal neurons. Based on a network mathematical model, Tewari and Parpura (2013) suggest that astrocytes modulate neuronal 
activity by introducing a delta rhythm in the CA1 pyramidal neurons. Repeated stimulations of astrocytes yield high-frequency oscillations of CA1 pyramidal neurons which is mainly based on the activation of NR1/NR2B subunits of the extra-synaptic NMDA receptors, which is in line with experimental results on epileptic seizures.

The topology of astrocytic networks brings another relevant aspect to the in silico models. Postnov et al. (2009) suggest a relatively simple way to create the geometry of astrocytic syncytia. Their results show a local $\mathrm{Ca}^{2+}$ spread in the astrocyte network and a possible acceleration of noise-induced $\mathrm{Ca}^{2+}$ waves.

Moreover, astrocytic ATP suppresses synaptic activity. Räisänen, Lenk and Hyttinen combined the spiking neuronal network model by Lenk (2011), the presynapse-astrocyte interface by De Pittà et al. (2011) and the astrocyte topology model by Lallouette et al. (2014). The combined stochastic model INEXA (Lenk et al., 2016) is to our knowledge the first neuron-astrocyte network model where one astrocyte is connected to several hundreds of excitatory synapses and up to five neighboring astrocytes. The authors showed that with increasing ratio of astrocytes in the network, the neuronal activity becomes more synchronous.

The neuron-astrocyte network can also be described with a mean field theoretical approach (Sotero and Martínez-Cancino 2010). External stimuli consisting of single spikes or spike trains separately applied to the presynaptic neurons and the astrocytes revealed complex patterns due to the astrocytic influence. Thus, in this model, the activation of astrocytes alone leads to a response of the neuronal network. Even without external stimuli, spontaneous astrocytic $\mathrm{Ca}^{2+}$ oscillations can result in excitation of the neuronal activity in Sotero and Martínez-Cancino (2010).

Yang and Yeo (2015) developed a conceptual model of the whole human nervous by considering the sensorial pathway from sensory neurons to neurons to astrocytes. Their conceptual model is divided in four layers: (1) sensory neurons, (2) neurons in the brain that are connected (3) to astrocytes which (4) form an astrocyte network. The model is able to switch from amplitude modulation (below $15 \mathrm{~Hz}$ ) to frequency modulation (between 15 and $35 \mathrm{~Hz}$ ). Increasing the number of stimuli leads to $\mathrm{Ca}^{2+}$ waves spreading through the entire astrocyte domain.

\section{Further model applications}

Beyond computational modelling of biological systems, astrocytes were also included to extend the classical artificial neural networks used in classification methods. Porto-Pazos et al. used astrocytes to induce synaptic potentiation at single synapses of artificial neural networks. This improved the performance of neural networks on classification problems (Alvarellos-González et al., 2012; Porto-Pazos et al., 2011). A further extension of this bio-inspired machine learning approach is a fully automatized and co-evolutionary learning process, which avoids the manual parameter tuning for each specific problem (Mesejo et al., 2015). Plans to incorporate such artificial neuron-astrocyte networks in the context of deep learning networks are currently considered (Pastur-Romay et al., 2016).

Important is also the role of astrocytes in pathological conditions. Astrocytes are considered as key players in pathologies like epilepsy or Huntington's and Alzheimer's disease. Therefore, in recent years the number of computational models investigating the role of astrocytes in these diseases has increased. For example, astrocytes modulate synaptic transmission and neural excitability and by that induce synchronous firing of neurons, which is associated with epilepsy (Volman et al. 2012; Amiri et al. 2013; Ullah et al. 2009; Tewari and Parpura 2013). In Alzheimer's disease, the gliotransmitter GABA is released in greater amounts if the astrocytes are in a pathological state - so-called reactive reactive astrocytes (Haim et al., 2015). Such healthy and the pathological conditions were simulated using the INEXA model (see above) through a stepwise increase of the astrocytic rate of GABA release (Lenk, 2011). With low GABA release rates, activity reduction is light and homogeneously distributed over the neurons. With large release rates though, a subgroup of neurons becomes totally silent, which reduces the overall network activity and in turn contributes to silence further neurons. In Alzheimer's disease, this could correspond to e.g. memory impairment.

Wade and colleagues (2012) investigated the potential implication of astrocytes in self-repair mechanisms. Retrograde signaling via astrocytic endocannabinoids enhances the neurotransmitter release probability at damaged synapses. Thus, in this model, the bidirectional communication between astrocytes and neurons and the resulting astrocytic control of synaptic activity leads to a self-repair of faulty synapses (Wade et al. 2012).

\section{Conclusions}

Computational models of single astrocytes, astrocyte networks and astrocyte-neuron networks contribute to a better understanding of astrocyte function. Since astrocytes have been intensively investigated decades later 
then neurons, naturally, their current models fall far behind neuronal models in terms of cell morphology and cell biophysics in 3D. Existing computational models either reproduce thus contribute to explain experimental data or they can be used as an extension of experiments, elucidating aspects of the system which are not directly accessible to experimental methods. In addition, computational models are not only developed in order to understand the physiological, but also the pathological behaviour of astrocytes. Thus, the role of astrocytes in diseases like epilepsy or Alzheimer's disease has started to be investigated with the help of computational models. Moreover, artificial models of astrocytes may increase the performance of artificial neural networks in classical classification problems.

Thus, so far the function of astrocytes in neuronal networks was investigated by means of a wide range of approaches. However, in order to get a clearer view of the impact of astrocytes on neuronal information processing, collaboration between experimental and computational approaches is more indispensable than ever.

\section{References}

Allam, S.L., Ghaderi, V.S., Bouteiller, J.-M.C., Legendre, A., Ambert, N., Greget, R., Bischoff, S., Baudry, M., Berger, T.W., 2012. A Computational Model to Investigate Astrocytic Glutamate Uptake Influence on Synaptic Transmission and Neuronal Spiking. Front. Comput. Neurosci. 6, 1-16. doi:10.3389/fncom.2012.00070

Alvarellos-González, A., Pazos, A., Porto-Pazos, A.B., 2012. Computational models of neuron-astrocyte interactions lead to improved efficacy in the performance of neural networks. Comput. Math. Methods Med. 2012, 476324. doi:10.1155/2012/476324

Amiri, M., Hosseinmardi, N., Bahrami, F., Janahmadi, M., 2013. Astrocyte-neuron interaction as a mechanism responsible for generation of neural synchrony: a study based on modeling and experiments. J. Comput. Neurosci. 34, 489-504. doi:10.1007/s10827-012-0432-6

Aubert, A., Costalat, R., 2005. Interaction between astrocytes and neurons studied using a mathematical model of compartmentalized energy metabolism. J. Cereb. Blood Flow Metab. 25, 1476-1490. doi:10.1038/sj.jcbfm 9600144

Bellinger, S., 2005. Modeling calcium wave oscillations in astrocytes. Neurocomputing 65-66, 843-850. doi:10.1016/j.neucom.2004.10.081

Bezprozvanny, 1lya, Watras, J., Ehrlich, B.E., 1991. Bell-shaped calcium-response curves of lns(1,4,5)P3and calcium-gated channels from endoplasmic reticulum of cerebellum. Nature 351, 751-754. doi: $10.1038 / 351751 \mathrm{a} 0$

Cao, P., Donovan, G., Falcke, M., Sneyd, J., 2013. A stochastic model of calcium puffs based on singlechannel data. Biophys. J. 105, 1133-1142. doi:10.1016/j.bpj.2013.07.034

Cao, P., Tan, X., Donovan, G., Sanderson, M.J., Sneyd, J., 2014. A Deterministic Model Predicts the Properties of Stochastic Calcium Oscillations in Airway Smooth Muscle Cells. PLoS Comput. Biol. 10. doi:10.1371/journal.pcbi.1003783

Chander, B.S., Chakravarthy, V.S., 2012. A Computational Model of Neuro-Glio-Vascular Loop Interactions. PLoS One 7, 1-11. doi:10.1371/journal.pone.0048802

Dallérac, G., Chever, O., Rouach, N., 2013. How do astrocytes shape synaptic transmission? Insights from electrophysiology. Front. Cell. Neurosci. 7, 159. doi:10.3389/fncel.2013.00159

De Pittà, M., Goldberg, M., Volman, V., Berry, H., Ben-Jacob, E., 2009. Glutamate regulation of calcium and IP3 oscillating and pulsating dynamics in astrocytes. J. Biol. Phys. 35, 383-411. doi:10.1007/s10867-009-9155-y

De Pittà, M., Volman, V., Berry, H., Parpura, V., Volterra, A., Ben-Jacob, E., 2012. Computational quest for understanding the role of astrocyte signaling in synaptic transmission and plasticity. Front. Comput. Neurosci. 6, 1-25. doi:10.3389/fncom.2012.00098

De Young, G.W., Keizer, J., 1992. A single-pool inositol 1,4,5-trisphosphate-receptor-based model for agonist-stimulated oscillations in Ca2+ concentration. Proc. Natl. Acad. Sci. U. S. A. 89, 9895-9.

Di Castro, M.A., Chuquet, J., Liaudet, N., Bhaukaurally, K., Santello, M., Bouvier, D., Tiret, P., Volterra, A., 2011. Local Ca2+ detection and modulation of synaptic release by astrocytes. Nat. Neurosci. 14, $1276-$ 1284. doi:10.1038/nn.2929

Diamond, J.S., 2005. Deriving the glutamate clearance time course from transporter currents in CA1 hippocampal astrocytes: transmitter uptake gets faster during development. J Neurosci 25, 2906-2916. doi:10.1523/JNEUROSCI.5125-04.2005

Dupont, G., Goldbeter, A., 1993. One-pool model for Ca2+ oscillations involving Ca2+ and inositol 1,4,5- 
trisphosphate as co-agonists for Ca2+ release. Cell Calcium 14, 311-322. doi:10.1016/01434160(93)90052-8

Edwards, J.R., Gibson, W.G., 2010. A model for Ca2+ waves in networks of glial cells incorporating both intercellular and extracellular communication pathways. J. Theor. Biol. 263, 45-58. doi:10.1016/j.jtbi.2009.12.002

Giaume, C., Koulakoff, A., Roux, L., Holcman, D., Rouach, N., 2010. Astroglial networks: a step further in neuroglial and gliovascular interactions. Nat Rev Neurosci 11, 87-99. doi:10.1038/nrn2757

Goldberg, M., De Pittà, M., Volman, V., Berry, H., Ben-Jacob, E., 2010. Nonlinear gap junctions enable long-distance propagation of pulsating calcium waves in astrocyte networks. PLoS Comput. Biol. 6, e1000909. doi:10.1371/journal.pcbi.1000909

Goldbeter, A., Dupont, G., Berridge, M.J., 1990. Minimal model for signal-induced Ca2+ oscillations and for their frequency encoding through protein phosphorylation. Proc. Natl. Acad. Sci. U. S. A. 87, 14611465. doi:10.1073/pnas.87.4.1461

Gómez-Gonzalo, M., Navarrete, M., Perea, G., Covelo, A., Martín-Fernández, M., Shigemoto, R., Luján, R., Araque, A., 2015. Endocannabinoids induce lateral long-term potentiation of transmitter release by stimulation of gliotransmission. Cereb. Cortex 25, 3699-3712. doi:10.1093/cercor/bhu231

Goto, I., Kinoshita, S., Natsume, K., 2004. The model of glutamate-induced intracellular Ca2+ oscillation and intercellular Ca2+ wave in brain astrocytes. Neurocomputing 58, 461-467. doi:10.1016/j.neucom.2004.01.082

Haim, L. Ben, Sauvage, M.C., Ceyzériat, K., Curtin, J.F., 2015. Elusive roles for reactive astrocytes in neurodegenerative diseases. Front. Cell. Neurosci. 9, 1-27. doi:10.3389/fncel.2015.00278

Henneberger, C., Papouin, T., Oliet, S., Rusakov, D., 2010. Long-term potentiation depends on release of D serine from astrocytes. Nature 463, 232-236. doi:10.1038/nature08673

Höfer, T., Venance, L., Giaume, C., 2002. Control and plasticity of intercellular calcium waves in astrocytes: a modeling approach. J. Neurosci. 22, 4850-4859. doi:20026493

Iacobas, D.A., Suadicani, S.O., Spray, D.C., Scemes, E., 2006. A stochastic two-dimensional model of intercellular Ca2+ wave spread in glia. Biophys. J. 90, 24-41. doi:10.1529/biophysj.105.064378

Jolivet, R., Magistretti, P.J., Weber, B., 2009. Deciphering neuron-glia compartmentalization in cortical energy metabolism. Front Neuroenergetics 1, 1-10. doi:10.3389/neuro.14.004.2009

Kager, H., Wadman, W.J., Somjen, G.G., 2007. Seizure-like afterdischarges simulated in a model neuron. J. Comput. Neurosci. 22, 105-128. doi:10.1007/s10827-006-0001-y

Kager, H., Wadman, W.J., Somjen, G.G., 2002. Conditions for the triggering of spreading depression studied with computer simulations. J. Neurophysiol. 88, 2700-12. doi:10.1152/jn.00237.2002

Kager, H., Wadman, W.J., Somjen, G.G., 2000. Simulated seizures and spreading depression in a neuron model incorporating interstitial space and ion concentrations. J. Neurophysiol. 84, 495-512. doi:10.1016/0165-3806(89)90085-0

Kang, M., Othmer, H.G., 2009. Spatiotemporal characteristics of calcium dynamics in astrocytes. Chaos 19, 1-21. doi:10.1063/1.3206698

Kimelberg, H., Nedergaard, M., 2010. Functions of astrocytes and their potential as therapeutic targets. Neurotherapeutics 7, 338-353. doi:10.1016/j.nurt.2010.07.006.Functions

Kinney, J.P., Spacek, J., Bartol, T.M., Bajaj, C.L., Harris, K.M., Sejnowski, T.J., 2013. Extracellular sheets and tunnels modulate glutamate diffusion in hippocampal neuropil. J. Comp. Neurol. 521, 448-464. doi:10.1002/cne.23181

Lallouette, J., De Pittà, M., Ben-Jacob, E., Berry, H., 2014. Sparse short-distance connections enhance calcium wave propagation in a 3D model of astrocyte networks. Front. Comput. Neurosci. 8, 1-18. doi: $10.3389 /$ fncom.2014.00045

Larter, R., Craig, M.G., 2005. Glutamate-induced glutamate release: A proposed mechanism for calcium bursting in astrocytes. Cit. Chaos 15, 1-11. doi:10.1063/1.2102467.

Lenk, K., 2011. A simple phenomenological neuronal model with inhibitory and excitatory synapses, in: Travieso-González, C.M., Alonso-Hernández, J.B. (Eds.), Proceedings of the 5th International Conference on Advances in Nonlinear Speech Processing, NOLISP'11. Springer-Verlag, Berlin, Heidelberg, pp. 232-238.

Lenk, K., Räisänen, E., Hyttinen, J.A.K., 2016. Understanding the role of astrocytic GABA in simulated neural networks. Proc. IEEE EMBC 6121-6124.

Li, B., Chen, S., Zeng, S., Luo, Q., Li, P., 2012. Modeling the Contributions of Ca2+ Flows to Spontaneous $\mathrm{Ca} 2+$ Oscillations and Cortical Spreading Depression-Triggered Ca2+ Waves in Astrocyte Networks. PLoS One 7, e48534. doi:10.1371/journal.pone.0048534 
Li, Y.-X., Rinzel, J., 1994. Equations for InsP3 Receptor-mediated [Ca2+]i Oscillations Derived from a Detailed Kinetic Model: A Hodgkin-Huxley Like Formalism. J. Theor. Biol. 166, 461-473. doi:10.1006/jtbi.1994.1041

Linne, M.-L., Jalonen, T.O., 2014. Astrocyte-neuron interactions: from experimental research-based models to translational medicine, 1st ed, Progress in molecular biology and translational science. Elsevier Inc. doi:10.1016/B978-0-12-397897-4.00005-X

Matrosov, V. V., Kazantsev, V.B., 2011. Bifurcation mechanisms of regular and chaotic network signaling in brain astrocytes. Chaos 21. doi:10.1063/1.3574031

McIver, S.R., Faideau, M., Haydon, P.G., 2013. Neural-Immune Interactions in Brain Function and Alcohol Related Disorders. Springer US, Boston, MA. doi:10.1007/978-1-4614-4729-0

Mesejo, P., Ibáñez, O., Fernández-Blanco, E., Cedrón, F., Pazos, A., Porto-Pazos, A.B., 2015. Artificial neuron-glia networks learning approach based on cooperative coevolution. Int. J. Neural Syst. 25, 1550012. doi:10.1142/S0129065715500124

Mesiti, F., Veleti, M., Floor, P.A., Balasingham, I., 2015. Astrocyte-neuron communication as cascade of equivalent circuits. Nano Commun. Netw. 6, 183-197. doi:10.1016/j.nancom.2015.08.005

Nadkarni, S., Jung, P., 2007. Modeling synaptic transmission of the tripartite synapse. Phys. Biol. 4, 1-9. doi:10.1088/1478-3975/4/1/001

Nadkarni, S., Jung, P., 2004. Dressed neurons: modeling neural-glial interactions. Phys. Biol. 1, 35-41. doi: $10.1088 / 1478-3967 / 1 / 1 / 004$

Nadkarni, S., Jung, P., 2003. Spontaneous oscillations of dressed neurons: a new mechanism for epilepsy? Phys. Rev. Lett. 91, 268101. doi:10.1103/PhysRevLett.91.268101

Nadkarni, S., Jung, P., Levine, H., 2008. Astrocytes optimize the synaptic transmission of information. PLoS Comput. Biol. 4, e1000088. doi:10.1371/journal.pcbi.1000088

Naeem, M., McDaid, L.J., Harkin, J., Wade, J.J., Marsland, J., 2015. On the Role of Astroglial Syncytia in Self-Repairing Spiking Neural Networks. IEEE Trans. Neural Networks Learn. Syst. 26, 2370-2380. doi:10.1109/TNNLS.2014.2382334

Øyehaug, L., Østby, I., Lloyd, C.M., Omholt, S.W., Einevoll, G.T., 2012. Dependence of spontaneous neuronal firing and depolarisation block on astroglial membrane transport mechanisms. J. Comput. Neurosci. 32, 147-165. doi:10.1007/s10827-011-0345-9

Paixão, S., Klein, R., 2010. Neuron-astrocyte communication and synaptic plasticity. Curr. Opin. Neurobiol. 20, 466-473. doi:10.1016/j.conb.2010.04.008

Pasti, L., Zonta, M., Pozzan, T., Vicini, S., Carmignoto, G., 2001. Cytosolic calcium oscillations in astrocytes may regulate exocytotic release of glutamate. J. Neurosci. 21, 477-484. doi:21/2/477 [pii]

Pastur-Romay, L.A., Cedrón, F., Pazos, A., Porto-Pazos, A.B., 2016. Deep artificial neural networks and neuromorphic chips for big data analysis: Pharmaceutical and bioinformatics applications. Int. J. Mol. Sci. 17, 1-26. doi:10.3390/ijms 17081313

Perea, G., Araque, A., 2010. GLIA modulates synaptic transmission. Brain Res. Rev. 63, 93-102. doi:10.1016/j.brainresrev.2009.10.005

Perea, G., Araque, A., 2005. Properties of synaptically evoked astrocyte calcium signal reveal synaptic information processing by astrocytes. J. Neurosci. 25, 2192-2203. doi:10.1523/JNEUROSCI.396504.2005

Petravicz, J., Fiacco, T. a, McCarthy, K.D., 2008. Loss of IP3 receptor-dependent Ca2+ increases in hippocampal astrocytes does not affect baseline CA1 pyramidal neuron synaptic activity. J. Neurosci. 28, 4967-4973. doi:10.1523/JNEUROSCI.5572-07.2008

Pinsky, P.F., Rinzel, J., 1994. Intrinsic and network rhythmogenesis in a reduced traub model for CA3 neurons. J. Comput. Neurosci. 1, 39-60. doi:10.1007/BF00962717

Porto-Pazos, A.B., Veiguela, N., Mesejo, P., Navarrete, M., Alvarellos, A., Ibáñez, O., Pazos, A., Araque, A., 2011. Artificial astrocytes improve neural network performance. PLoS One 6, 1-8. doi:10.1371/journal.pone.0019109

Postnov, D.E., Koreshkov, R.N., Brazhe, N.A., Brazhe, A.R., Sosnovtseva, O. V., 2009. Dynamical patterns of calcium signaling in a functional model of neuron-astrocyte networks. J. Biol. Phys. 35, 425-445. doi:10.1007/s10867-009-9156-x

Postnov, D.E., Ryazanova, L.S., Brazhe, N.A., Brazhe, A.R., Maximov, G. V., Mosekilde, E., Sosnovtseva, O. V., 2008. Giant glial cell: New insight through mechanism-based modeling. J. Biol. Phys. 34, 441457. doi:10.1007/s10867-008-9070-7

Rusakov, D.A., 2001. The Role of Perisynaptic Glial Sheaths in Glutamate Spillover and Extracellular Ca2+ Depletion. Biophys. J. 81, 1947-1959. doi:10.1016/S0006-3495(01)75846-8 
Sahlender, D.A., Savtchouk, I., Volterra, A., 2014. What do we know about gliotransmitter release from astrocytes? Philos. Trans. R. Soc. Lond. B. Biol. Sci. 369, 20130592. doi:10.1098/rstb.2013.0592

Savtchenko, L.P., Rusakov, D.A., 2014. Regulation of rhythm genesis by volume-limited, astroglia-like signals in neural networks. Philos. Trans. R. Soc. B Biol. Sci. 369, 20130614-20130614. doi:10.1098/rstb.2013.0614

Savtchenko, L.P., Sylantyev, S., Rusakov, D.A., 2013. Central synapses release a resource-efficient amount of glutamate. Nat. Neurosci. 16, 10-2. doi:10.1038/nn.3285

Scemes, E., Giaume, C., 2006. Astrocyte CalciumWaves: What They Are and What They Do. Glia 54, 716725. doi:10.1002/glia

Shigetomi, E., Tong, X., Kwan, K.Y., Corey, D.P., Khakh, B.S., 2012. TRPA1 channels regulate astrocyte resting calcium and inhibitory synapse efficacy through GAT-3. Nat. Neurosci. 15, 70-80. doi:10.1038/nn.3000

Siekmann, I., Wagner, L.E., Yule, D., Crampin, E.J., Sneyd, J., 2012. A kinetic model for type i and II IP3R accounting for mode changes. Biophys. J. 103, 658-668. doi:10.1016/j.bpj.2012.07.016

Somjen, G.G., Kager, H., Wadman, W.J., 2008. Computer simulations of neuron-glia interactions mediated by ion flux. J. Comput. Neurosci. 25, 349-365. doi:10.1007/s10827-008-0083-9

Sotero, R.C., Martínez-Cancino, R., 2010. Dynamical mean field model of a neural-glial mass. Neural Comput. 22, 969-997. doi:10.1162/neco.2009.04-09-1002

Srinivasan, R., Huang, B.S., Venugopal, S., Johnston, A.D., Chai, H., Zeng, H., Golshani, P., Khakh, B.S., 2015. $\mathrm{Ca}(2+)$ signaling in astrocytes from Ip3r2(-/-) mice in brain slices and during startle responses in vivo. Nat. Neurosci. 18, 708-17. doi:10.1038/nn.4001

Tewari, S., Parpura, V., 2013. A possible role of astrocytes in contextual memory retrieval: An analysis obtained using a quantitative framework. Front. Comput. Neurosci. 7, 145. doi: $10.3389 /$ fncom. 2013.00145

Tewari, S.G., Majumdar, K.K., 2012. A mathematical model of the tripartite synapse: astrocyte-induced synaptic plasticity. J. Biol. Phys. 38, 465-496. doi:10.1007/s10867-012-9267-7

Traub, R.D., Wong, R.K., Miles, R., Michelson, H., 1991. A model of a CA3 hippocampal pyramidal neuron incorporating voltage-clamp data on intrinsic conductances. J. Neurophysiol. 66, 635-650.

Ullah, G., Cressman, J.R., Barreto, E., Schiff, S.J., 2009. The influence of sodium and potassium dynamics on excitability, seizures, and the stability of persistent states: II. Network and glial dynamics. J. Comput. Neurosci. 26, 171-183. doi:10.1007/s10827-008-0132-4

Ullah, G., Jung, P., Cornell-Bell, A.H., 2006. Anti-phase calcium oscillations in astrocytes via inositol (1, 4, 5)-trisphosphate regeneration. Cell Calcium 39, 197-208. doi:10.1016/j.ceca.2005.10.009

Venance, L., Stella, N., Glowinski, J., Giaume, C., 1997. Mechanism involved in initiation and propagation of receptor-induced intercellular calcium signaling in cultured rat astrocytes. J. Neurosci. 17, 1981-92.

Vincent, A.J., Gasperini, R., Foa, L., Small, D.H., 2010. Astrocytes in Alzheimer's disease: Emerging roles in calcium dysregulation and synaptic plasticity. J. Alzheimer's Dis. 22, 699-714. doi:10.3233/JAD2010-101089

Volman, V., Bazhenov, M., Sejnowski, T.J., 2012. Computational models of neuron-astrocyte interaction in epilepsy. Front. Comput. Neurosci. 6, 58. doi:10.3389/fncom.2012.00058

Volman, V., Ben-Jacob, E., Levine, H., 2007. The Astrocyte as a Gatekeeper of Synaptic Information Transfer. Neural Comput. 326, 303-326.

Wade, J., McDaid, L., Harkin, J., Crunelli, V., Kelso, S., 2012. Self-repair in a bidirectionally coupled astrocyte-neuron (AN) system based on retrograde signaling. Front. Comput. Neurosci. 6, 76. doi: $10.3389 /$ fncom. 2012.00076

Wade, J.J., McDaid, L.J., Harkin, J., Crunelli, V., Kelso, J. a S., 2011. Bidirectional coupling between astrocytes and neurons mediates learning and dynamic coordination in the brain: A multiple modeling approach. PLoS One 6,1-24. doi:10.1371/journal.pone.0029445

Wei, F., Shuai, J., 2011. Intercellular calcium waves in glial cells with bistable dynamics. Phys. Biol. 8, 26009. doi:10.1088/1478-3975/8/2/026009

Yang, Y., Yeo, C.K., 2015. Conceptual NetworkModel From Sensory Neurons to Astrocytes of the Human Nervous System. IEEE Trans. Biomed. Eng. 62, 1843-1852.

Zeng, S.S., Li, B., Zeng, S.S., Chen, S., 2009. Simulation of Spontaneous Ca2+ Oscillations in Astrocytes Mediated by Voltage-Gated Calcium Channels. Biophys. J. 97, 2429-2437. doi:10.1016/j.bpj.2009.08.030

Zheng, K., Scimemi, A., Rusakov, D.A., 2008. Receptor actions of synaptically released glutamate: the role of transporters on the scale from nanometers to microns. Biophys. J. 95, 4584-4596. 
doi:10.1529/biophysj.108.129874

Zheng, Y., Pan, Y., Harris, S., Billings, S., Coca, D., Berwick, J., Jones, M., Kennerley, A., Johnston, D., Martin, C., Devonshire, I.M., Mayhew, J., 2010. A dynamic model of neurovascular coupling:

Implications for blood vessel dilation and constriction. Neuroimage 52, 1135-1147.

doi:10.1016/j.neuroimage.2010.01.102

Zorec, R., Araque, A., Carmignoto, G., Haydon, P.G., Verkhratsky, A., Parpura, V., 2012. As a Signalling Route. ASN Neuro 4, 103-119. doi:10.1042/AN20110061

\section{Acknowledgement:}

The authors thank Antonio Ladron De Guevara Ruiz for providing Figure 1.

\section{Funding:}

FO's and KO's work was supported by the Deutsche Forschungsgemeinschaft [GRK 1589 "Sensory Computation in Neural Systems"]. KL's work was supported by the 3DNeuroN project in the European Union's Seventh Framework Programme, Future and Emerging Technologies [grant agreement number 296590] and Tekes funded Human Spare Part project. 